Rabaska

Revue d'ethnologie de l'Amérique française

DUBÉ, PHILIPPE, DAVID KAREL et PHILIPPE BAYLAUCQ. Marcel Baril. Figure énigmatique de l'art québécois. Sainte-Foy, Les Presses de l'Université Laval, 2002, 287 p. ISBN 2-7637-7648-5

\title{
Valérie Rousseau
}

Volume 3, 2005

URI : https://id.erudit.org/iderudit/201722ar

DOI : https://doi.org/10.7202/201722ar

Aller au sommaire du numéro

Éditeur(s)

Société québécoise d'ethnologie

ISSN

1703-7433 (imprimé)

1916-7350 (numérique)

Découvrir la revue

Citer ce compte rendu

Rousseau, V. (2005). Compte rendu de [DUBÉ, PHILIPPE, DAVID KAREL et PHILIPPE BAYLAUCQ. Marcel Baril. Figure énigmatique de l'art québécois.

Sainte-Foy, Les Presses de l'Université Laval, 2002, 287 p. ISBN 2-7637-7648-5].

Rabaska, 3, 139-144. https://doi.org/10.7202/201722ar d'utilisation que vous pouvez consulter en ligne. 
Dubé, Philippe, David Karel et Philippe Baylauce. Marcel Baril. Figure énigmatique de l'art québécois. Sainte-Foy, Les Presses de l'Université Laval, 2002, 287 p. ISBN 2-7637-7648-5.

Cette publication apporte un éclairage fondamental sur l'œuvre et le cheminement de cet artiste méconnu, originaire de Warwick dans la région des Bois-Francs au Québec. Elle s'inscrit dans la suite logique de la reconnaissance tardive et indispensable de son œuvre, quelques années avant son décès en 1999 à l'âge de 82 ans; à ce titre citons notamment l'exposition Mais qui est donc Marcel Baril ? qui se tenait au Musée de Charlevoix en 1995, regroupant 105 tableaux et objets personnels, et le film documentaire Mystère $B$. réalisé par Philippe Baylaucq en 1997. Par le biais des études et des reproductions de qualité qui le composent, l'ouvrage dresse un bilan de l'œuvre laissé par Marcel Baril, quant à ses références, ses sources, son ancrage historique et ses significations. Comme l'écrivent les auteurs - le cinéaste Philippe Baylaucq, l'historien d'art David Karel et l'ethnologue Philippe Dubé - dans la présentation de ce livre, l'objectif consiste à lever le voile sur cet artiste incontournable, mais dont l'œuvre, inclassable et personnel, réalisé dans le retrait, demeure méconnu de la scène des arts visuels au Québec. 
Dans la préface de l'ouvrage qu'elle signe, Lise Bissonnette (présidentedirectrice générale, Bibliothèque nationale du Québec) évoque les souvenirs d'une première rencontre qu'elle eut avec Marcel Baril en 1996, durant la rétrospective de ses œuvres au Marché Bonsecours, et d'une seconde à son atelier de l'avenue de Versailles à Paris un an plus tard, ville où il avait élu domicile permanent depuis 1954. D'entrée de jeu, le texte de Lise Bissonnette situe l'atmosphère de mystère et de candeur lucide qui se dégage du personnage et de ses œuvres, insistant sur la nécessité d'explorer l'une de ces figures de «l'autre modernité » en art au Québec. Comme elle l'avance à juste titre, Marcel Baril, en individualiste, « frôle presque toutes les " écoles" de son siècle et leur échappe aussitôt, toujours poussé par ses propres fièvres. La fenêtre ouverte ici sur " l'autre peinture", celle des inclassables indépendants qui, avant et après la deuxième guerre mondiale, ont rompu avec l'académisme sans renier l'humain qu'on appelle toujours avec un certain mépris " la figure ", est d'un passionnant enseignement ».

Dans l'étude respective qu'ils livrent et qui constitue le corps principal de la publication, Philippe Baylaucq, David Karel et Philippe Dubé situent la contribution artistique de Marcel Baril, permettant d'en épouser la portée et le rayonnement. Leur analyse, complémentaire dans leur approche, s'appuie sur l'examen de plus de 300 tableaux de signature distincte et aux sujets variés, et sur le dépouillement de fonds d'archives - notamment le Fonds Marcel Baril constitué de correspondance s'échelonnant de 1938 à 1979, d'une douzaine de manuscrits et de dessins -, ajoutant au caractère inédit de cette recherche substantielle.

Le premier texte, essentiel, s'appuie sur une démarche de type ethnographique. Philippe Baylaucq réalise une sélection d'extraits tirés d'entrevues qu'il a menées à compter de 1994 auprès de Marcel Baril, à l'occasion de la préparation du film documentaire qu'il lui consacrera. Son texte fournit une vue d'ensemble de l'être paradoxal qu'était Marcel Baril. L'approche de l'auteur, sensible et nuancée, qui traduit son attachement pour l'artiste, tente de cerner les mécanismes qui rythment et structurent sa pensée créatrice. Marcel Baril se livre généreusement, après cinquante années de silence à œuvrer en marge des réseaux artistiques et, comme le note l'auteur, après « l'unique exposition parisienne de la Galerie R. Creuze » au printemps 1950. Ses propos sont captés dans l'urgence, après sa sortie de l'hôpital, où l'on tente de le soigner d'une maladie qui pourrait lui être fatale. " Les paroles de peintre », organisées par Philippe Baylaucq, révèlent un Marcel Baril observateur, insatiable et « flâneur 》, imprégné de la nécessité de créer et de ses souvenirs d'enfance, comme autant de « motifs » obsessionnels (nudité, seins, Pierrot, mort) qui traversent sa production artistique : «Pourquoi la peinture ? [...] Quand j'étais tout petit, j'ai pris un crayon et j'ai commencé 
à griffonner sur du papier, et j'ai continué. [...] Je faisais ça comme je respirais, explique Marcel Baril, dont la jeunesse a pourtant été marquée par des problèmes respiratoires. [...] Il n'y a jamais eu un commencement à ça. Je n'ai jamais dit: " un jour je vais devenir peintre ". Je respire, et, comme je respire, je dessine mes rêves. [...] L'écriture, la littérature, il aurait fallu apprendre, tandis que la peinture je n'avais pas besoin d'apprendre. [...] Tout le monde a des souvenirs. Moi, j'ai une partie de mon cerveau que j'ai habituée à transformer les choses en images. [...] Petit, j'étais souvent malade avec mon asthme et, comme je manquais souvent l'école, je me retrouvais souvent seul dans mon propre monde et je dessinais. Et en quelque sorte j'ai continué à ma façon toute ma vie. » Cette compilation préparée par Philippe Baylaucq, dans laquelle on déplore seulement l'absence de légendes de certaines photos d'archives, ouvre à une multiplicité de lectures, pénétrant avec subtilité les contradictions de l'artiste et ses moments de solitude forcée et souhaitée : "Je me suis aperçu alors que, lorsque je peins, je m'adresse toujours à quelqu'un. [...] Mais pourquoi exposer ?, demandait Marcel Baril aux étudiants de la Cité Universitaire où il travailla longtemps à titre de secrétaire à la Maison des étudiants canadiens. Quand on peint on a un besoin de communication et vous me donnez tout ça, je n'ai pas besoin d'aller chercher ailleurs. "

«C'est incroyable de ne rien savoir comme ça, mais c'est mieux. Je découvre tout seul. [...] Je dois à chaque fois chercher tout seul les solutions, des solutions à moi. Enfin, il ne faut pas savoir les choses, il faut les trouver tout seul. [...] Je ne veux surtout pas me servir de ce qu'on apprend dans les livres et les écoles. ") Les contradictions de Marcel Baril traversent aussi l'étude suivante, celle de David Karel, qui situe le travail de l'artiste parmi les grands courants artistiques du XX $\mathrm{XX}^{\mathrm{e}}$ siècle et cerne son ancrage au Québec par une analyse approfondie de l'iconographie et de la grammaire stylistique de son œuvre. Sous le titre évocateur « Le refus tranquille », l'article de David Karel rappelle, dans un premier temps, « les circonstances et les volontés qui ont conduit le jeune Marcel Baril à un point de rupture spirituelle et esthétique ", afin de montrer ensuite la "singularité de l'homme et ses réalisations ». Devant le peu de sources documentaires sur Marcel Baril, David Karel se rabat sur la subjectivité du peintre, puisant tour à tour dans les sujets traités, dans les intentions révélées, dans le système philosophique auquel il adhère (le personnalisme), et dans les sources artistiques dont il s'imprègne. Dans le premier sous-chapitre intitulé "L'éveil », il arrive de perdre le fil conducteur en raison des ellipses, du choix des termes séparateurs et des allers-retours dans le temps. Cette difficulté est renforcée par l'interprétation personnelle donnée de certaines cuvres, mais aussi par la lecture faite de sa manière. Comme se le demande David Karel, la facture de la peinture de 
Marcel Baril est-elle vraiment neutre ? De même, Baril est-il réellement réticent à aborder la question du sacré dans son art - si l'on se réfère aux cuvres reproduites dans le présent ouvrage - ou le fait-il par le biais d'un registre inventé plus subtil, notamment dans l'attitude de repli et dans le caractère d'intériorité qu'ont les personnages ? Le texte de David Karel s'érige avec force à partir du second sous-chapitre, «L'apprentissage », lorsqu'il identifie les groupes d'appartenance de l'artiste: « Aucune des multiples facettes du style barilien ne domine. De Marcel Baril on ne peut dire qu'il est seulement onirique, ou réaliste, ou naïf, ou expressionniste, ou même érotique ; il a déjà été tout cela et davantage ! [...] De toute manière, il n'a jamais été un suiveur. Et l'on peut dire aussi, puisque manifestement et peutêtre bien malgré lui il a goûté à l'art de son temps, qu'il accuse par moments des influences des plus variées. Farouchement indépendant, indifférent au gain et résolu à se préserver de l'opinion critique, Marcel Baril aura tracé une voie comme en butinant, passant par des repères tantôt familiers et tantôt inconnus. » Outre l'influence de trois modernismes (surréalisme, expressionnisme, néo-primitivisme) qui marquent à certains égards sa production avant son départ pour Paris, David Karel fait état de l'importance des enseignements qu'il reçut durant un an (1934) de l'artiste Rodolphe Duguay, alors qu'adolescent il fréquentait le séminaire de Nicolet. Rodolphe Duguay continuera de le suivre durant sa première année à l'École des beaux-arts de Montréal (1937). « L'intérêt que vous me portez, lui écrira Marcel Baril, est mon unique soutien. » David Karel soulève également l'intérêt manifeste de l'artiste pour le modernisme figuratif durant ses études, s'attarde à ses sources bibliographiques, décelant qu'aucun «volume de sa bibliothèque ne porte sur l'abstraction », et relève l'admiration fidèle et soutenue qu'avait pour lui François Hertel. Les parallèles qu'il tisse entre l'œuvre de Marcel Baril et ceux d'autres artistes, notamment Francis Cruber, James Ensor et Max Beckmann, sont convaincants, de même que son argumentation visant à démontrer la singularité et la persévérance de Marcel Baril, dans la distance qu'il prend par rapport aux avant-gardes du $\mathrm{XX}^{\mathrm{e}}$ siècle.

L'étude de David Karel, dans laquelle il est question de la cohérence de l'œuvre de Marcel Baril, de même que de la richesse de ses fonctions visuelles et littéraires, opère un lien logique avec la suivante, celle de Philippe Dubé, qui s'attache à révéler le peintre-chroniqueur qu'il fut, par une approche empruntée à l'ethnologie. Par une présentation rigoureuse largement documentée, Philippe Dubé réussit à « topographier l'environnement culturel dans lequel Marcel Baril s'est déployé, identifier et questionner son entourage, situer ses lieux d'ancrage et, bien entendu, mettre en rapport son parcours avec les traces - ses cuvres - qu'il a bien voulu nous livrer ». Comme Dubé le précise d'emblée, pour appuyer sa problématique, Marcel Baril a toujours 
opéré comme un narrateur - constat que soulève également dans leur texte Philippe Baylaucq et David Karel - et que confie l'artiste lui-même, lorsqu'il avoue : « Dans mes peintures, il y a plein de souvenirs de lieux et de gens qui ne sont plus et que je fais revenir comme ça. [...] Il y a une chose qui est sûre, c'est que, dans tout ce qui est là, il y a une lutte contre la mort. Essayer de sauver les souvenirs. [...] J'essaie de sauver les meubles de mon naufrage. » Des illustrations qu'il créait alors qu'il était enfant, et dont l'imagerie puise ses sources dans les journaux, en passant par ses ambitions prématurées d'éditeur (il n'a que 12 ans lorsqu'il imprime son deuxième journal La Nature en 1929) et jusqu'aux récits picturaux qu'il livre plus tard dans sa peinture, Marcel Baril exprime ses ambitions de mémorialiste par un souci de transmission et un besoin de raconter. Les différents registres que l'on trouve régulièrement à l'intérieur de chaque composition invitent d'ailleurs à la narration et à l'échelonnement d'une histoire réelle ou fictive. Dans une formulation heureuse, Philippe Dubé dira que Marcel Baril «a accédé à la parole par la peinture et nous a entretenus depuis son plus jeune âge des choses de la vie. Ici son texte devenu texture prend la forme d'un discours intime qu'il nous livre par capsules, comme si chaque tableau avait sa part à dire. [...] Quand il est face à lui-même, il se fait le fidèle rapporteur/reporteur de ses souvenirs, de ses émotions, de ses angoisses et, pourquoi pas, de ses élucubrations. " L'auteur, avec force détails, compile tour à tour les écrits sur l'art et les institutions artistiques qui émergent du contexte culturel et intellectuel foisonnant dans lequel naît Marcel Baril, passe en revue le récit familial de l'artiste, soulève sa maîtrise précoce de la caricature et de l'illustration, rapporte les fréquentations qui marquèrent son cheminement d'homme et d'artiste (dont François Hertel), et parcourt le territoire de l'intime en jeu dans ses œuvres. Philippe Dubé tente enfin de saisir la position atypique qu'a prise Marcel Baril par rapport à l'art de son temps, nous permettant de mieux comprendre les circonstances de son exil à Paris et d'expliquer la périphérie qu'il a occupée à ce jour dans une certaine histoire de l'art. Sur ce dernier point, Philippe Dubé propose à juste titre que les inflexions de Marcel Baril pour le réel et son appartenance populaire, comme le revendiquent les artistes associés au réalisme dans leur quête de vérité et d'authenticité, permettent d'expliquer sa grammaire stylistique qui puise dans l'art dit populaire, naiff et primitif.

Suite à la postface signée par l'historienne d'art Claudette Hould, et au cours de laquelle elle livre un témoignage succinct et personnel de Marcel Baril alors qu'il était en fonction à la Maison des étudiants canadiens, les auteurs ont compilé, dans la première partie de la bibliographie, les différents fonds d'archives et articles consultés dans le cadre de leur recherche, se rapportant spécifiquement à Marcel Baril. Il aurait été intéressant, pour mieux 
mesurer la réception critique de l'œuvre de l'artiste, que cette section soit clairement distincte de la bibliographie générale, où il semble être question d'ouvrages et de documents généraux sur le contexte artistique et culturel. La chronologie comparée, placée à la fin de la publication, pertinente dans le cadre de cette étude, permet le recoupement de deux moments : le premier trace l'évolution du cheminement artistique de Marcel Baril, suivi, année après année, de l'actualité artistique de l'époque. Ce deuxième moment, secondaire bien que judicieux, aurait pu être réduit au profit des faits relevant de la démarche et de l'œuvre de Marcel Baril. La liste des œuvres reproduites permet de localiser activement celles auxquelles les auteurs, principalement David Karel et Philippe Dubé, font référence à maintes reprises. Ce support contribue largement à la clarté des textes et à la lisibilité de cet œuvre dense et éclectique. À l'inverse d'une présentation sobre, la conception visuelle de l'ouvrage véhicule un certain dynamisme ; l'utilisation de couleurs vives, d'une typographie soutenue pour les nombreuses citations, du soulignement coloré et de signes graphiques, ne permet pas toujours d'alléger les différents niveaux d'informations sur le plan graphique.

Enfin, le retranchement dans lequel s'est placé Marcel Baril et qui constitue l'élément moteur du présent ouvrage, renvoie à l'essai du philosophe et critique d'art Jean-Yves Jouannais, intitulé Artistes sans æuvres. I would prefer not to, consacré aux vies obscures d'artistes qui traversèrent l'histoire de l'art et la vie intellectuelle sur la pointe des pieds mais non sans influence ; ils animèrent les uns - Michel Foucault rêva d'écrire l'anthologie d'existences anonymes après son Histoire de la folie, et Jean Dubuffet s'activa à la découverte de l'art brut et de ses artistes récalcitrants - et poursuivirent les autres - que l'on pense à cette étrange figure qu'était Jacques Vaché, écrivain, et par laquelle André Breton sera profondément séduit. « Le silence est un muscle », écrit Jean-Yves Jouannais, se référant à Marcel Duchamp et à son art du peu. «Une multitude de productions n'accèdent pas à la lumière, ajoutet-il encore. [...] Leurs messages ne sont pas destinés vers l'extérieur, ils fonctionnent, au contraire en usage interne, comme autant [...] d'exercices destinés à contrer l'agnosie, à retarder l'altération de la mémoire. » Marcel Baril a œuvré avec passion, par besoin, dans une sorte de « banlieue de la postérité », comme s'il s'agissait d'un gage d'indépendance et d'équilibre. L'éclairage qu'apporte soudainement et avec beaucoup de substance cet ouvrage sur Marcel Baril n'en dissipe pas moins le mystère; au contraire, il demeure entier, alimenté dans les fondements même de la figure de l'artiste, furtive et investie de nos projections multiples. 